\title{
Basin of Attraction through Invariant Curves and Dominant Functions
}

\author{
Ziyad AlSharawi, ${ }^{1}$ Asma Al-Ghassani, ${ }^{1}$ and A. M. Amleh ${ }^{2}$ \\ ${ }^{1}$ Department of Mathematics and Statistics, Sultan Qaboos University, P.O. Box 36, Alkhoud, 123 Muscat, Oman \\ ${ }^{2}$ Sciences and Engineering, Paris-Sorbonne University Abu Dhabi, P.O. Box 38044, Abu Dhabi, UAE
}

Correspondence should be addressed to Ziyad AlSharawi; alshalzm@alsharawi.info

Received 9 February 2015; Accepted 6 May 2015

Academic Editor: Garyfalos Papashinopoulos

Copyright (C) 2015 Ziyad AlSharawi et al. This is an open access article distributed under the Creative Commons Attribution License, which permits unrestricted use, distribution, and reproduction in any medium, provided the original work is properly cited.

We study a second-order difference equation of the form $z_{n+1}=z_{n} F\left(z_{n-1}\right)+h$, where both $F(z)$ and $z F(z)$ are decreasing. We consider a set of invariant curves at $h=1$ and use it to characterize the behaviour of solutions when $h>1$ and when $0<h<1$. The case $h>1$ is related to the Y2K problem. For $0<h<1$, we study the stability of the equilibrium solutions and find an invariant region where solutions are attracted to the stable equilibrium. In particular, for certain range of the parameters, a subset of the basin of attraction of the stable equilibrium is achieved by bounding positive solutions using the iteration of dominant functions with attracting equilibria.

\section{Introduction}

Second-order difference equations of the form

$$
z_{n+1}=z_{n} F\left(z_{n-1}\right), \quad n \in \mathbb{N}:=\mathbb{Z}^{+} \cup\{0\},
$$

where $F(z)$ is a continuous function, have been widely used in applications [1-4]. Several models in mathematical biology take the form of (1) under the assumptions that $F(z)$ is decreasing and $z F(z)$ is bounded and increasing $[4,5]$. A well-known example is Pielou's difference equation which has been suggested to model the growth of a single species with delayed-density dependence $[4,6]$. In Pielou's equation, $F$ takes the form $F(z)=\lambda /(1+\alpha z)$. Adding or subtracting a constant $h$ from (1) can be interpreted mathematically as a perturbation of the model, or biologically as constant stocking or constant yield harvesting [7-9]. These meaningful applications motivate investigating the dynamics of the difference equation

$$
z_{n+1}=z_{n} F\left(z_{n-1}\right) \pm h, \quad n \in \mathbb{N}, h>0 .
$$

However, when the functions $F(z)$ and $z F(z)$ are both decreasing, the corresponding difference equation becomes more abstract and so far little work has been done to investigate its dynamics. Although our ultimate goal is to reach a general theory for this type of difference equation, we find it interesting to consider $F(z)=b /(-1+z)$ as a prototype, and so we focus this work on the dynamics of the equation

$$
\begin{aligned}
z_{n+1}= & z_{n} F\left(z_{n-1}\right)+h, \\
F(z) & =\frac{b}{-1+z}, \\
& n \in \mathbb{N}, h, b>0 .
\end{aligned}
$$

Among the important aspects of solutions of a difference equation are boundedness and global stability. On several occasions, the question of boundedness of all solutions of a particular difference equation was settled by finding invariant curves. Invariant curves of a second-order difference equation are plane curves on which forward orbits that start on a curve remain on the curve. Finding invariant curves, studying their properties and their relation with Liapunov functions is an active area of research [10-13].

In recent years, several results that give sufficient conditions for global stability or global attractivity of equilibrium solutions of difference equations have been established. Most of the results rely on the monotonicity of the function 
defined by the difference equation under investigation; see, for instance, $[1,11,14,15]$ and the references therein. Another approach of establishing global attractivity is to relate solutions of the difference equation to the solutions of a onedimensional map where (under some conditions) the global attractivity of the equilibrium of the map implies the global attractivity of the equilibrium of the difference equation. This technique, which is sometimes called enveloping or dominance, is well-known in first-order difference equations [16], and it has been extended recently to tackle some higher order difference equations $[17,18]$.

In this paper, we focus on (3), where $b, h>0$ and the initial conditions are restricted to assure the existence of positive solutions (persistent solutions). Throughout our work, solutions are meant to be well-defined solutions. We consider a set of invariant curves and verify certain inequalities they satisfy. The inequalities describe the movement of solutions in the phase plane, which are ultimately used to prove that the larger positive equilibrium of (3) is a global attractor for a certain range of the parameter $h$. When $h>1$, (3) is related (via a change of variables) to the $\mathrm{Y} 2 \mathrm{~K}$ difference equation where the global attractivity of its positive equilibrium was an open problem for several years till it was settled recently by Merino [19]. Also, the case $h=1$ is related (via a change of variables) to Lyness equation in which solutions remain on invariant curves. The case $0<h<1$ of (3) exhibits very interesting dynamics and is discussed in Section 3.

\section{The Case $h>1$}

In this section, we consider (3) and assume $h>1$. Thus, throughout this section, we refer to the equation

$$
z_{n+1}=z_{n} F\left(z_{n-1}\right)+h=\frac{b z_{n}}{-1+z_{n-1}}+h,
$$

$$
b>0, h>1 \text {. }
$$

The substitutions

$$
\begin{aligned}
z_{n} & =\frac{1}{p-q}\left(q x_{n}+p\right), \\
b & =\frac{q^{2}}{p-q} \\
h & =\frac{p}{p-q}
\end{aligned}
$$

transform (4) to

$$
x_{n+1}=\frac{p+q x_{n}}{1+x_{n-1}}, \quad x_{-1}, x_{0} \geq 0, n \in \mathbb{N} .
$$

Equation (6) is known in the literature as the $\mathrm{Y} 2 \mathrm{~K}$ problem [18]. Observe that, for $b>0$ and $h>1$, we have $z_{n}>h$ if and only if $x_{n}>0$. Also, the restriction $b>0, h>1$ on the $(b, h)$-parameters is equivalent to the restriction $0<q<p$ on the $(p, q)$-parameters. In $(6)$, it is obvious that positive initial conditions give rise to positive solutions, and there exists a unique positive equilibrium. Proving that the positive equilibrium is a global attractor with respect to the positive quadrant was an open question for over a decade till it was settled by Merino [19]. Merino transformed (6) into

$$
y_{n+1}=\frac{p / q^{2}+y_{n}}{1 / q+y_{n-1}}, \quad y_{-1}, y_{0}>0, n \in \mathbb{N},
$$

where $q y_{n}=x_{n}$. Then he used the function

$$
\begin{aligned}
I_{n} & :=I\left(y_{n-1}, y_{n}\right) \\
& =\left(1+\frac{1}{y_{n-1}}\right)\left(1+\frac{1}{y_{n}}\right)\left(u^{2}-u+y_{n-1}+y_{n}\right),
\end{aligned}
$$

to prove the next crucial lemma, where $u$ is the positive equilibrium of (7), which is given by

$$
\bar{y}=u=\frac{1}{2 q}\left(q-1+\sqrt{(q-1)^{2}+4 p}\right) .
$$

Lemma 1 (see [19]). Consider $y_{n-1}, y_{n}>0$, and then either $I_{n+1} \leq I_{n}$ or $I_{n+2} \leq I_{n}$.

Merino used this crucial lemma to show that the positive equilibrium of (7) is a global attractor. However, the given proof of Lemma 1 depends heavily on Mathematica code. So here, we present an alternative proof of Lemma 1, which is more trackable. But in order to keep things within the context of our work, we translate the curves $I_{n}$ of (7) to the curves $J_{n}$ of (4), which are given by

$$
\begin{aligned}
J_{n} & :=J\left(z_{n-1}, z_{n}\right)=\left(1+\frac{b}{z_{n-1}-h}\right)\left(1+\frac{b}{z_{n}-h}\right) \\
& \cdot\left(-h+(1-h) B+z_{n-1}+z_{n}\right),
\end{aligned}
$$

where $B=(1 / b)\left(\bar{z}_{2}-h\right)$ and $\bar{z}_{2}$ is the large equilibrium of (4). Observe that at $h=1, J\left(z_{n-1}, z_{n}\right)=$ constant is in fact an invariant curve for Lyness equation after a transformation.

For typographical reasons, we define $x:=z_{-1}, y:=z_{0}$, and $z:=z_{1}$ in (4). Without further mention, we consider $(x, y)$ to be in the region $\mathscr{R}=\{(s, t): s, t>h\}$, which is in fact the positive quadrant of (6). We find

$$
\begin{aligned}
& J_{n+1}-J_{n} \\
& =J\left(y, \frac{b y}{x-1}+h\right)-J(x, y) \\
& =\frac{-(h-1)(y-h+b)}{(y-h) y(x-1)(x-h)}(b y-f(x))(y-g(x)),
\end{aligned}
$$

where $f(x)=(x-1)(x-h)$ and $g(x)=B(x-1)$. Because $h>1$ and $b>0$, we have $J_{n+1}>J_{n}$ whenever

$$
\min \left\{g(x), \frac{1}{b} f(x)\right\}<y<\max \left\{g(x), \frac{1}{b} f(x)\right\},
$$

while $J_{n+1} \leq J_{n}$ otherwise. It is worth mentioning here that if $\left(x_{-1}, x_{0}\right)=(x, y)$ is located in the region between $g(x)$ and $(1 / b) f(x)$, then the point $\left(x_{0}, x_{1}\right)=(y, z)=(y, b y /(x-1)+h)$ is guaranteed to be out of the region. Now, we are in a position to give our trackable proof of Lemma 1 translated in terms of $J_{n}$. 
Proof. We consider $J_{n+1}>J_{n}$ and show that $J_{n+2} \leq J_{n}$. Because

$$
J_{n+2}-J_{n}=\left(J_{n+1}-J_{n}\right)\left(\frac{-\left(J_{n+1}-J_{n+2}\right)}{\left(J_{n+1}-J_{n}\right)}+1\right),
$$

then we need to show that

$$
\frac{\left(J_{n+1}-J_{n+2}\right)}{\left(J_{n+1}-J_{n}\right)} \geq 1 .
$$

We write

$$
\begin{aligned}
& \frac{\left(J_{n+1}-J_{n+2}\right)}{\left(J_{n+1}-J_{n}\right)} \\
& =\frac{J(y, z)-J(z, b z /(y-1)+h)}{J(y, b y /(x-1)+h)-J(x, y)} \\
& =\frac{y(x-1)(x-h)(z-h+b)[z-g(y)][b z-f(y)]}{z(y-1)(z-h)(y-h+b)[y-g(x)][f(x)-b y]} .
\end{aligned}
$$

Our aim here is to show that the R.H.S. is larger than or equal to 1 . We proceed by taking two cases, namely, the following.

Case 1. Consider

$$
(x, y) \in \mathscr{R}_{r}:=\left\{(s, t): s>\bar{z}_{2}, g(s)<t<\frac{1}{b} f(s)\right\} .
$$

Case 2. Consider

$$
\begin{aligned}
(x, y) & \in \mathscr{R}_{\ell} \\
& :=\left\{(s, t): h<s<\bar{z}_{2}, \frac{1}{b} f(s)<t<g(s)\right\} .
\end{aligned}
$$

Case 1. Observe that the conditions we have on $x, y$, and $z$ force all factors in the numerator and denominator to be positive. Furthermore, we have $y>x$ and the fact that $y<$ $(1 / b) f(x)$ gives $z<x$. Next, write (15) as

$$
\begin{aligned}
\frac{\left(J_{n+1}-J_{n+2}\right)}{\left(J_{n+1}-J_{n}\right)}= & \frac{y}{z} \cdot \frac{(1-b z / f(y))}{(1-b y / f(x))} \cdot \frac{(1+b /(z-h))}{(1+b /(y-h))} \\
& \cdot \frac{(g(y)-z)}{(y-g(x))}
\end{aligned}
$$

and observe that each one of the first three factors in the R.H.S. is larger than 1. Thus, we obtain

$$
\frac{\left(J_{n+1}-J_{n+2}\right)}{\left(J_{n+1}-J_{n}\right)}>\frac{(g(y)-z)}{(y-g(x))} .
$$

Use Figure 1(a) to conclude that

$$
g(y)-z>y-z>y-x>y-g(x) .
$$

So, the R.H.S. of Inq. (19) is larger than one which completes the proof of Case 1.
Case 2. In this case, we have $x>y$, and since $z=b y /(x-1)+$ $h$, we obtain $z>x$. To make all factors in both the numerator and denominator of (15) positives, we rewrite the equation as

$$
\begin{aligned}
& \frac{\left(J_{n+1}-J_{n+2}\right)}{\left(J_{n+1}-J_{n}\right)} \\
& =\frac{y(x-1)(x-h)(z-h+b)[z-g(y)][b z-f(y)]}{z(y-1)(z-h)(y-h+b)[g(x)-y][b y-f(x)]} .
\end{aligned}
$$

Again we show that the R.H.S. is larger than 1. To achieve this task, we rewrite (21) as

$$
\begin{aligned}
\frac{\left(J_{n+1}-J_{n+2}\right)}{\left(J_{n+1}-J_{n}\right)}= & \frac{(x-1)}{(y-1)} \\
& \cdot \frac{(x-h)(z-h+b)(z-g(y))}{(z-h)(y-h+b)(g(x)-y)} \\
& \cdot \frac{[b-f(y) / z]}{[b-f(x) / y]} .
\end{aligned}
$$

Observe that the first and last factors are larger than 1, and therefore, we obtain

$$
\frac{\left(J_{n+1}-J_{n+2}\right)}{\left(J_{n+1}-J_{n}\right)}>\frac{(x-h)(z-h+b)(z-g(y))}{(z-h)(y-h+b)(g(x)-y)} .
$$

Since $g(y)<y$ and $g(x)<x$ (see Figure $1(\mathrm{~b})$ ), we obtain

$$
\frac{\left(J_{n+1}-J_{n+2}\right)}{\left(J_{n+1}-J_{n}\right)}>\frac{(x-h)(z-h+b)(z-y)}{(z-h)(y-h+b)(x-y)} .
$$

Because the function

$$
G(z)=\frac{(z-h+b)(z-y)}{(z-h)}
$$

is increasing on the interval $[y, \infty)$ as long as $y \geq h$, then $z>x$ implies $G(z)>G(x)$. Therefore,

$$
\frac{\left(J_{n+1}-J_{n+2}\right)}{\left(J_{n+1}-J_{n}\right)}>\frac{(x-h+b)}{(y-h+b)}>1
$$

as required, which completes the proof.

\section{The Case $0<h<1$}

In this section, we consider (3) and let $0<h<1$. Thus, throughout this section, we refer to the equation

$$
z_{n+1}=z_{n} F\left(z_{n-1}\right)+h=\frac{b z_{n}}{-1+z_{n-1}}+h, \quad 0<h<1 .
$$

We investigate the stability of equilibrium solutions, the existence of periodic solutions, and the boundedness and persistence of solutions. Throughout our work, we use stability to denote local stability and persistence to denote positive solutions. We also find an invariant region and give a range of the parameters for which the region is part of the basin of attraction of the stable equilibrium. 


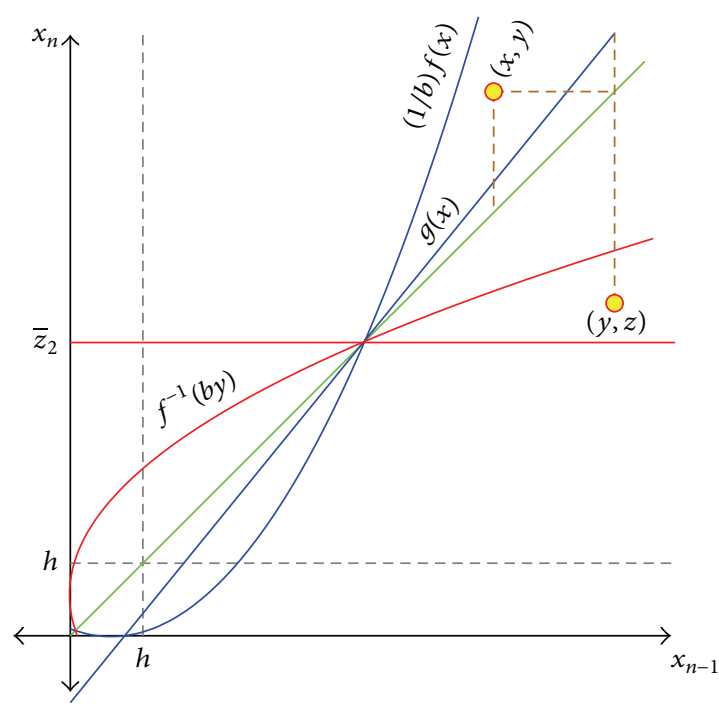

(a)

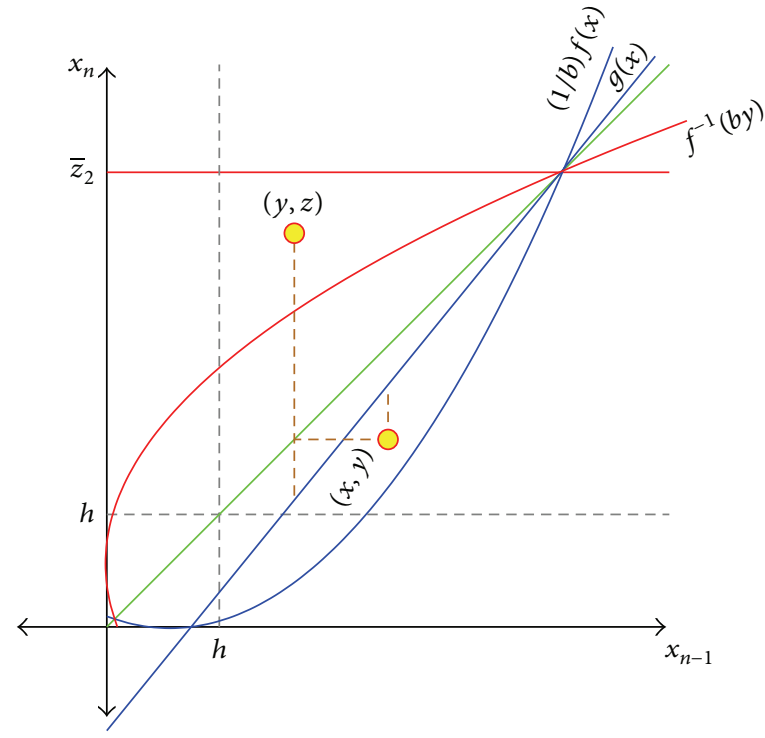

(b)

Figure 1: (a) clarifies the proof of Case 1 while (b) clarifies the proof of Case 2. The scale on the axes is intentionally missing because the graphs represent the general situation.

3.1. Stability of Equilibria and Periodic Solutions. The two equilibrium points $\bar{z}_{1}, \bar{z}_{2}$ of (27) satisfy $0<\bar{z}_{1}<h$ and $\bar{z}_{2}>1$; furthermore, they are increasing in $h$ and satisfy $\bar{z}_{1} \bar{z}_{2}=h$. The linearized form of (27) at $\bar{z}_{2}$ is given by

$$
u_{n+2}-F\left(\bar{z}_{2}\right) u_{n+1}-\bar{z}_{2} F^{\prime}\left(\bar{z}_{2}\right) u_{n}=0 .
$$

Because

$$
\begin{aligned}
-F\left(\bar{z}_{2}\right) & =-\frac{\bar{z}_{2}-h}{\bar{z}_{2}}=-1+\frac{h}{\bar{z}_{2}}=\bar{z}_{1}-1, \\
-\bar{z}_{2} F^{\prime}\left(\bar{z}_{2}\right) & =\frac{\bar{z}_{2}-h}{\bar{z}_{2}-1}=1+\frac{1-h}{\bar{z}_{2}-1},
\end{aligned}
$$

then

$$
\begin{aligned}
&-1<-F\left(\bar{z}_{2}\right)<0, \\
&-\bar{z}_{2} F^{\prime}\left(\bar{z}_{2}\right)>1 .
\end{aligned}
$$

Thus, the eigenvalues of the linearized equation at $\bar{z}_{2}$ are complex and out of the unit circle which make $\bar{z}_{2}$ a repeller. Similarly, we find the eigenvalues of the linearized equation at $\bar{z}_{1}$. We have

$$
\begin{aligned}
& M:=-F\left(\bar{z}_{1}\right)=-\frac{\bar{z}_{1}-h}{\bar{z}_{1}}=-1+\frac{h}{\bar{z}_{1}}=\bar{z}_{2}-1, \\
& N:=-\bar{z}_{1} F^{\prime}\left(\bar{z}_{1}\right)=\frac{\bar{z}_{1}-h}{\bar{z}_{1}-1}=1-\frac{1-h}{1-\bar{z}_{1}} .
\end{aligned}
$$

Hence, $M>0$ and $0<N<1$, which give us that $\bar{z}_{1}$ is either locally stable or a saddle. Figure 2 illustrates this case in the parameter space.

Before we address the issue of boundedness, let us have a look at the possibility of periodic solutions. We always use

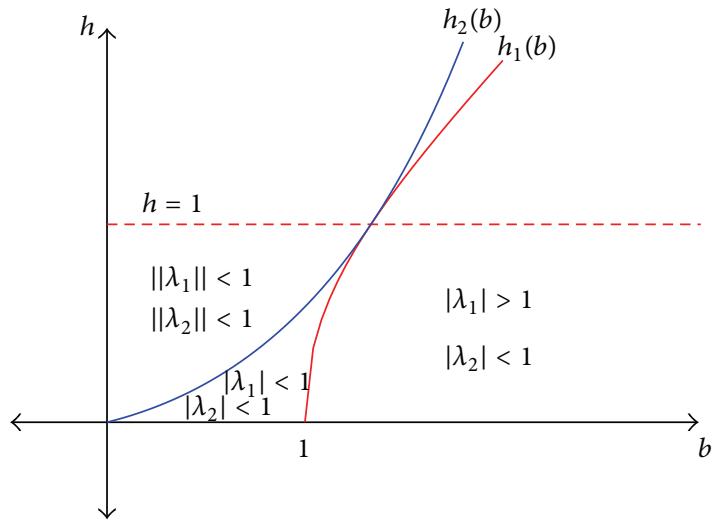

Figure 2: This figure illustrates the magnitude of the eigenvalues associated with $\bar{z}_{1}$ in the $(b, h)$-plane. $\|\cdot\|$ denotes the magnitude when the eigenvalue is nonreal, while $|\cdot|$ denotes the magnitude when the eigenvalue is real. $h_{1}(b)=1-b+2 \sqrt{b(b-1)}$ comes from the condition $N=M-1$, and $h_{2}(b)=b(4+3 b) / 4(4-b)$ comes from the condition $M^{2}-4 N=0$.

period to denote the prime period. We start with period-two solutions by considering the equations

$$
\begin{aligned}
& (\phi-h)(\phi-1)-b \psi=0, \\
& (\psi-h)(\psi-1)-b \phi=0 .
\end{aligned}
$$

Observe that the two parabolas intersect in either two or four points and $\bar{z}_{1}, \bar{z}_{2}$ must be among them. We substitute and eliminate the factor that gives $\bar{z}_{1}$ and $\bar{z}_{2}$ to obtain

$$
\phi^{2}-(h+1-b) \phi+(b-1)(b-h)=0 .
$$


This equation has two real solutions when $(h+1-b)^{2}>4(b-$ $1)(b-h)$, which gives the region to the left of the curve $h_{1}(b)$ in Figure 2. So, suppose $(h+1-b)^{2}>4(b-1)(b-h)$ and name the period-two solution $\{\bar{\phi}, \bar{\psi}\}$. If we go back to the case $h>1$, we find that $\min \{\bar{\phi}, \bar{\psi}\}<h$, which is obviously not in the region of global stability considered in [19]. For $0<h<1$, it is possible that $\min \{\bar{\phi}, \bar{\psi}\}<0$ while $\max \{\bar{\phi}, \bar{\psi}\}>0$; however this is beyond our interest in this paper. On the other hand, it is possible that $\min \{\bar{\phi}, \bar{\psi}\}>0$. In fact, this takes place when $0<b<1$ and $h \geq b$, or when $b \geq 1$ and $h_{1}$ (b) $\leq h \leq b$. We formalize this discussion in the following result.

Proposition 2. Let $0<h<1$ and $b \leq(1 / 3)(h+1+$ $2 \sqrt{h^{2}-h+1}$ ). Equation (27) has period-two solution $\{\bar{\phi}, \bar{\psi}\}$ that satisfies one of the following:

(i) $\min \{\bar{\phi}, \bar{\psi}\} \geq 0$ and $\max \{\bar{\phi}, \bar{\psi}\} \leq h / b$ when $0<b \leq h$ or $h_{1}(b) \leq h \leq b$.

(ii) $\min \{\bar{\phi}, \bar{\psi}\}<0$ and $\max \{\bar{\phi}, \bar{\psi}\}>h / b$ when $h<b<1$.

Next, by algebraic manipulations of the equations

$$
\begin{aligned}
& z=y F(x)+h, \\
& x=z F(y)+h, \\
& y=x F(z)+h,
\end{aligned}
$$

we find that period-three solutions exist and can be positive. However, we avoid high level of computations and just give an interesting example of period-three solution that we use in the sequel. Let $h:=1-b(1-b)$ and consider $x_{-1}=0, x_{0}=$ $h$, we obtain a period-three solution given by $\{0, h, h(1-b)\}$, which is nonnegative whenever $b \leq 1$.

Finally, as a consequence of the boundedness and oscillation results that we establish later on, no periodic solutions of period higher than four exist. Thus, our discussion about the existence of periodic solutions ends by investigating the existence of period-four solutions. In fact, algebraic manipulations show that positive period-four solutions do not exist within the range of our parameters.

3.2. Boundedness of Nonnegative Solutions. In this section, we prove that the only positive solution of (27) that satisfies $z_{n}>$ 1 for all $n \geq-1$ is the equilibrium solution $\bar{z}_{2}$. Then we show that the remaining positive solutions are bounded. To achieve this task, we first establish a reversed form of the inequalities given in Lemma 1. We can follow the technique used to prove Lemma 1 (when $h>1$ ), but for the readers convenience, we give another elegant technique that serves as a handy tool for similar scenarios. We handle the expression $J_{n+2}-J_{n}$ using the points $(y, z)$. Thus, we need to consider

$$
\begin{aligned}
& T\left(\mathscr{R}_{r}\right)=\mathscr{R}_{r}^{*}, \\
& T\left(\mathscr{R}_{\ell}\right)=\mathscr{R}_{\ell}^{*},
\end{aligned}
$$

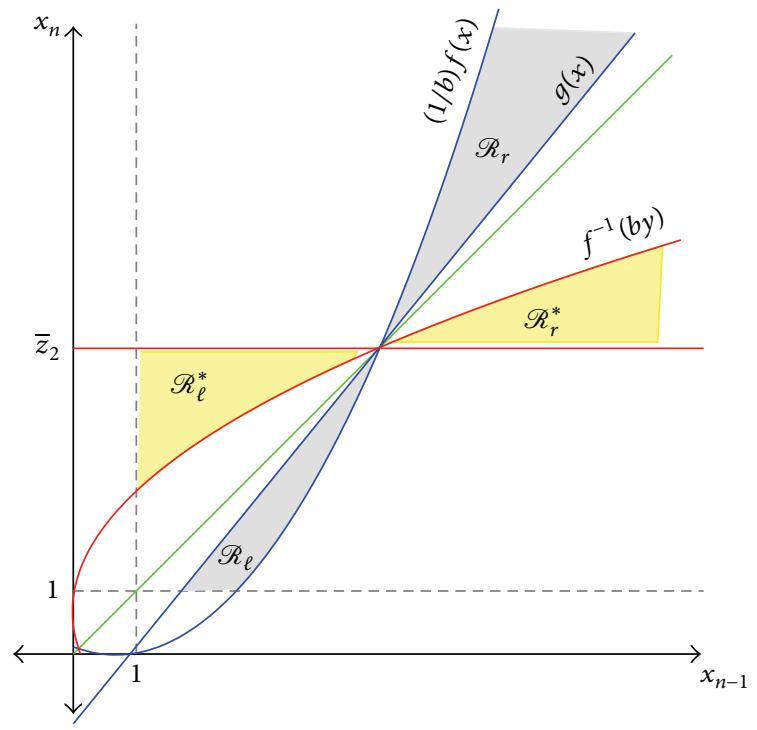

Figure 3: This figure illustrates the regions $\mathscr{R}_{\ell}, T\left(\mathscr{R}_{\ell}\right)=\mathscr{R}_{\ell}^{*}, \mathscr{R}_{r}$ and $T\left(\mathscr{R}_{r}\right)=\mathscr{R}_{r}^{*}$, where $0<h<1$.

where $T$ is the map defined by $T\left(x_{n-1}, x_{n}\right)=\left(x_{n}, x_{n+1}\right)$. We illustrate these sets in Figure 3, and it is straightforward to check that

$$
\mathscr{R}_{r}^{*}=\left\{(y, z): y>\bar{z}_{2}, \bar{z}_{2}<z<f^{-1}(b y)\right\}
$$

while

$$
\mathscr{R}_{\ell}^{*} \subset\left\{(y, z): 1<y<\bar{z}_{2}, f^{-1}(b y)<z<\bar{z}_{2}\right\} \text {. }
$$

Now, write

$$
\begin{aligned}
J_{n+2}-J_{n}= & J\left(x_{n+1}, x_{n+2}\right)-J\left(x_{n-1}, x_{n}\right) \\
= & {\left[J\left(z, \frac{b z}{y-1}+h\right)-J(y, z)\right] } \\
& +\left[J(y, z)-J\left(\frac{b y}{z-h}+1, y\right)\right],
\end{aligned}
$$

in which

$$
\begin{gathered}
J\left(z, \frac{b z}{y-1}+h\right)-J(y, z)=\frac{(h-1)}{(y-h)(z-h)} \\
\cdot \frac{(z-h+b)(b z-f(y))(g(y)-z)}{z(y-1)}, \\
J(y, z)-J\left(\frac{b y}{z-h}+1, y\right)=\frac{(h-1)}{(y-h)(z-h)} \\
\cdot \frac{(y-h+b)(b y-f(z))\left(z-\bar{z}_{2}\right)}{[b y-(h-1)(z-h)]} .
\end{gathered}
$$


Ignore the common factors and keep in mind that $(h-1)$ is negative and then define

$$
\begin{aligned}
Q_{1}(y, z) & =\lambda(f(y)-b z)(g(y)-z), \\
Q_{2}(y, z) & =-(b y-f(z))\left(z-\bar{z}_{2}\right), \\
\lambda & =\lambda(y, z) \\
& =\frac{(z-h+b)(b y-(h-1)(z-h))}{(y-h+b) z(y-1)} .
\end{aligned}
$$

Now, the following two basic results will be used in the sequel.

Proposition 3. Let $0<h<1$. Each of the following holds true.

(i) If $(y, z) \in \mathscr{R}_{r}^{*}$, then $\lambda \geq((z-h+b) /(y-h+b)) \cdot(y / z)$. $(b /(y-1))$; furthermore, if $b \geq h$ then $\lambda \geq b /(y-1)$.

(ii) If $(y, z) \in \mathscr{R}_{\ell}^{*}$, then $\lambda \geq 1 / B$.

Proof. (i) Because by $-(h-1)(z-h)>b y$, then we obtain the first part of the statement. To show the second part of the statement, we use the assumption $b \geq h$ and the fact that $y \geq$ $z \geq \bar{z}_{2}$ to conclude that

$$
\frac{(z-h+b)}{(y-h+b)} \cdot \frac{y}{z}=\frac{1-(h-b) / z}{1-(h-b) / y}>1,
$$

which completes the proof of part (i).

(ii) Because we have $\bar{z}_{2} \geq z \geq y>1$, then

$$
\begin{aligned}
\lambda & =\frac{(z-h+b)(b y-(h-1)(z-h))}{(y-h+b) z(y-1)} \\
& \geq \frac{(b y-(h-1)(z-h))}{z(y-1)} \\
& =\frac{(b y+(1-h)(z-h))}{b y} \cdot \frac{b y}{(y-1)} \cdot \frac{1}{z},
\end{aligned}
$$

and consequently,

$$
\lambda \geq \frac{b y}{(y-1)} \cdot \frac{1}{z} \geq \frac{b z}{(z-1)} \cdot \frac{1}{z}=\frac{b}{(z-1)} .
$$

Now, $z \leq \bar{z}_{2}$ implies $b /(z-1) \geq b /\left(\bar{z}_{2}-1\right)$, and hence

$$
\lambda \geq \frac{b}{\left(\bar{z}_{2}-h\right)}=\frac{1}{B} .
$$

Proposition 4. Let $(y, z) \in \mathscr{R}_{r}^{*}$ and consider $0<b<h<1$. Define

$$
\begin{aligned}
& A_{1}:=\lambda\left(y-1+\bar{z}_{2}-h\right), \\
& A_{2}:=\lambda(y-1)-b, \\
& A_{3}:=\bar{z}_{2}-b+B\left(\bar{z}_{2}-h\right) .
\end{aligned}
$$

Then $A_{1}$ and $A_{3}$ are positive; furthermore, $A_{2}+2 \sqrt{A_{1} A_{3}}>0$.
Proof. It is obvious that $A_{1}$ and $A_{3}$ are both positive. To show that $A_{2}+2 \sqrt{A_{1} A_{3}}>0$, we use part (i) of Proposition 3 to obtain

$$
\begin{aligned}
A_{2} & \geq b \frac{(z-h+b)}{(y-h+b)} \cdot \frac{y}{z}-b \geq b \frac{(z-h+b)}{z}-b \\
& \geq b \frac{\left(\bar{z}_{2}-h+b\right)}{\bar{z}_{2}}-b, \\
A_{1} & \geq \frac{(z-h+b)}{(y-h+b)} \cdot \frac{y}{z} \cdot \frac{b\left(y-1+\bar{z}_{2}-h\right)}{(y-1)} \\
& \geq \frac{b(z-h+b)}{z} \geq b \frac{\left(\bar{z}_{2}-h+b\right)}{\bar{z}_{2}} .
\end{aligned}
$$

Then, we obtain

$$
\begin{aligned}
A_{2}+ & 2 \sqrt{A_{1} A_{3}} \\
\geq & b \frac{\left(\bar{z}_{2}-h+b\right)}{\bar{z}_{2}}-b \\
& +2 \sqrt{b \frac{\left(\bar{z}_{2}-h+b\right)}{\bar{z}_{2}}} \sqrt{\bar{z}_{2}-b+B\left(\bar{z}_{2}-h\right)} .
\end{aligned}
$$

Because $0<b<h<1<\bar{z}_{2}$ and $q(t)=(t-h+b) / t$ is increasing in $t$, we obtain

$$
\begin{aligned}
A_{2}+ & 2 \sqrt{A_{1} A_{3}} \\
\geq & b \frac{(1-h+b)}{1}-b \\
& +2 \sqrt{b \frac{(1-h+b)}{1}} \sqrt{\bar{z}_{2}-b+B\left(\bar{z}_{2}-h\right)} \\
= & b(b-h)+2 \sqrt{b(1-h+b)} \sqrt{\bar{z}_{2}-b+B\left(\bar{z}_{2}-h\right)} \\
\geq & b(b-h)+2 b \sqrt{\bar{z}_{2}-b+B\left(\bar{z}_{2}-h\right)} .
\end{aligned}
$$

Finally, observe that

$$
\begin{aligned}
\bar{z}_{2}-b+B\left(\bar{z}_{2}-h\right) & \geq \bar{z}_{2}-b+B\left(\bar{z}_{2}-1\right)=2 \bar{z}_{2}-b \\
& >1
\end{aligned}
$$

which implies

$$
A_{2}+2 \sqrt{A_{1} A_{3}} \geq b(b-h)+2 b=b(h-b+2)>0 .
$$

Hence, the proof is complete.

Next, we proceed to show that either $J_{n+1} \geq J_{n}$ or $J_{n+2}>$ $J_{n}$. So, we consider $J_{n+1}<J_{n}$ and then show that $J_{n+2}>J_{n}$.

Lemma 5. Consider (27) with $0<h<1$. Let $\left\{z_{n}\right\}_{n=-1}^{\infty}$ be a nonequilibrium solution that satisfies $z_{n}>1$ for all $n \geq-1$. Either $J_{n+1} \geq J_{n}$ or $J_{n+2}>J_{n}$. 
Proof. Let $0<h<1$ and assume $J_{n+1}<J_{n}$. Because the common factor $(h-1)$ that we ignored in (39) is negative, it is sufficient to show that

$$
Q_{1}(y, z)+Q_{2}(y, z) \geq 0 \quad \forall(y, z) \in \mathscr{R}_{r}^{*} \cup \mathscr{R}_{\ell}^{*} .
$$

Again, we take two cases as follows.

Case 1. Let $(y, z) \in \mathscr{R}_{r}^{*}$. We transform $(y, z)$ into $(s, t)$ as follows: Let $g(y)-\bar{z}_{2}=s$ and $z-\bar{z}_{2}=(1-t) s$. We prove that $Q_{1}(y, z)+Q_{2}(y, z) \geq 0$ for all $0 \leq t \leq 1$ and $s \geq 0$. Because $g(y) \geq f^{-1}(b y)$ for all $y \geq \bar{z}_{2}$, then the $(s, t)$-region gives us more than $\mathscr{R}_{r}^{*}$. Use Taylor's expansion about $\left(\bar{z}_{2}, \bar{z}_{2}\right)$ to obtain

$$
\begin{aligned}
& Q_{1}(y, z)=\lambda\left[\left(2 \bar{z}_{2}-1-h\right) B Y^{2}\right. \\
& \left.\quad+\left(-3 \bar{z}_{2}+1+2 h\right) Y Z+b Z^{2}+B Y^{3}-Z Y^{2}\right], \\
& Q_{2}(y, z)=-b Y Z+\left(2 \bar{z}_{2}-1-h\right) Z^{2}+Z^{3},
\end{aligned}
$$

where $Y=y-\bar{z}_{2}$ and $Z=z-\bar{z}_{2}$, and then substitute $Y=y-\bar{z}_{2}=s / B$ and $Z=z-\bar{z}_{2}=(1-t) s$ to obtain after simplifications

$$
\begin{aligned}
& Q_{1}\left(\frac{s}{B}+\bar{z}_{2},(1-t) s+\bar{z}_{2}\right)=\lambda \frac{s^{2}}{B} \\
& \cdot t\left(\bar{z}_{2}+\frac{s}{B}-1+b B t\right), \\
& Q_{2}\left(\frac{s}{B}+\bar{z}_{2},(1-t) s+\bar{z}_{2}\right)=\frac{s^{2}}{B}(1-t) \\
& \cdot\left[-b+\left(2 \bar{z}_{2}-1-h\right) B(1-t)+s B(1-t)^{2}\right] .
\end{aligned}
$$

Ignore the positive common factors, and then our task will be to prove that

$$
\begin{aligned}
& \lambda t\left(\bar{z}_{2}+\frac{s}{B}-1+b B t\right)+(1-t) \\
& \quad \cdot\left[-b+\left(2 \bar{z}_{2}-1-h\right) B(1-t)+s B(1-t)^{2}\right]
\end{aligned}
$$

is positive for all $0 \leq t \leq 1$ and $s \geq 0$. Indeed, we rewrite the expression as

$$
\begin{aligned}
\lambda & \left(2 \bar{z}_{2}+\frac{s}{B}-h-1\right) t^{2} \\
& +\left(\bar{z}_{2}-b+B\left(\bar{z}_{2}-h\right)\right)(1-t)^{2} \\
& +\left(\lambda\left(\bar{z}_{2}+\frac{s}{B}-1\right)-b\right) t(1-t)+s B(1-t)^{3},
\end{aligned}
$$

and then based on the established notations and facts of Proposition 4, we have

$$
A_{1} t^{2}+A_{3}(1-t)^{2}+A_{2} t(1-t)+s B(1-t)^{3},
$$

and all terms of this expression are positives except possibly $A_{2} t(1-t)$. If $b \geq h$, then part (i) of Proposition 3 makes $A_{2} \geq$
0 . On the other hand, if $0<b<h<1$, then we can add and subtract $2 \sqrt{A_{1} A_{3}} t(1-t)$ and then write

$$
\begin{gathered}
A_{1} t^{2}+A_{3}(1-t)^{2}-2 \sqrt{A_{1} A_{3}} t(1-t) \\
=\left(\sqrt{A_{1}} t-\sqrt{A_{3}}(1-t)\right)^{2} .
\end{gathered}
$$

Next, use the fact that $A_{2}+2 \sqrt{A_{1} A_{3}} \geq 0$ from Proposition 4 to conclude that the expression is again positive. Hence, the proof of Case 1 is complete.

Case 2. Let $(y, z) \in \mathscr{R}_{\ell}^{*}$. Although the proof follows ideas of Case 1, the technicalities make the proof not obvious, which motivates us to write the complete proof here again. We make the transformations $\bar{z}_{2}-g^{-1}(y)=s$ and $\bar{z}_{2}-z=t s$ while $z-g^{-1}(y)=(1-t) s$. Thus, we obtain $\bar{z}_{2}-y=B s$. Again, we prove that $Q_{1}(y, z)+Q_{2}(y, z) \geq 0$ for all $0 \leq t \leq 1$ and $0 \leq s<\bar{z}_{2}$. Because $g^{-1}(y) \leq f^{-1}(b y)$ for all $1<y \leq \bar{z}_{2}$, then the $(s, t)$-region gives us more than $\mathscr{R}_{\ell}^{*}$. Write $Q_{1}(y, z)$ and $Q_{2}(y, z)$ as in Case 1 , and then

$$
\begin{aligned}
& Q_{1}\left(-s B+\bar{z}_{2},-s t+\bar{z}_{2}\right) \\
& \quad=B \lambda s^{2}\left(B^{2}-t\right)\left[\left(2 \bar{z}_{2}-1-h-s B\right)-\frac{b}{B} t\right], \\
& Q_{2}\left(-s B+\bar{z}_{2},-s t+\bar{z}_{2}\right) \\
& \quad=-s^{2} t\left[s t^{2}-\left(2 \bar{z}_{2}-1-h\right) t+\bar{z}_{2}-h\right] .
\end{aligned}
$$

Ignore the positive common factors and use the fact that $B \lambda \geq$ 1 and $B>1$, and then our task will be to prove that

$$
\begin{aligned}
Q(s, t)= & (1-t)\left[\left(2 \bar{z}_{2}-1-h-s B\right)-\frac{b}{B} t\right] \\
& -t\left[s t^{2}-\left(2 \bar{z}_{2}-1-h\right) t+\bar{z}_{2}-h\right]
\end{aligned}
$$

is positive for all $0 \leq t \leq 1$ and $0 \leq s<\bar{z}_{2}$. We manipulate the terms of $Q(s, t)$ and write it as

$$
Q(s, t)=\beta_{1}(1-t)^{2}+\beta_{2} t^{2},
$$

where

$$
\begin{aligned}
\beta_{1} & =\left[2 \bar{z}_{2}-h-B s-1+\left(\bar{z}_{2}-B s-1-\frac{b}{B}\right) t\right], \\
\beta_{2} & =\left[\bar{z}_{2}-B s-1+s(B-t)\right. \\
& \left.+\left(\bar{z}_{2}-B s-1-\frac{b}{B}\right)(1-t)\right] .
\end{aligned}
$$


Because $\bar{z}_{2}-B s=y$ and $g(y)=B(y-1)$, we write

$$
\begin{aligned}
\beta_{1} & =\left[\bar{z}_{2}-h+y-1+\left(y-1-\frac{b}{B}\right) t\right] \\
& =\frac{1}{B}\left[\bar{z}_{2}-b+(y-h) B+g(y) t+b(1-t)\right], \\
\beta_{2} & =\left[y-1+s(B-t)+\left(y-1-\frac{b}{B}\right)(1-t)\right] \\
& =\frac{1}{B}\left[y t+\left(g(y)+\bar{z}_{2}-b\right)(1-t)\right],
\end{aligned}
$$

and then we have $\beta_{1}, \beta_{2}>0$, and consequently we obtain $Q(s, t)$ positive, which completes the proof.

Lemma 6. A solution $\left\{z_{n}\right\}_{n=-1}^{\infty}$ of (27) that satisfies $z_{n}>1$ for all $n \geq-1$ must be bounded; furthermore, it must be bounded below by $1+\epsilon_{0}$ for some fixed $\epsilon_{0}>0$.

Proof. Recall that a point $\left(z_{-1}, z_{0}\right)=(x, y)$ is mapped to the point $\left(z_{0}, z_{1}\right)$ under the map $T$; that is, $T(x, y)=(y, b y /(x-$ $1)+h)$. We use the map $T$ to give a visualization of the set of initial points that we are interested in. For a given constant $c>1$, we have

$$
\begin{aligned}
L_{1} & :=\{(t, c): t>1\} \\
& \stackrel{T}{\rightarrow} L_{2}:=\left\{\left(c, \frac{b c}{t-1}+h\right): t>1\right\} .
\end{aligned}
$$

Next, we choose a suitable value for $c$ so that $T\left(T\left(L_{2}\right)\right)$ gets out of the region $x, y>1$. Observe that $L_{1}$ is a horizontal line segment while $L_{2}=T\left(L_{1}\right)$ is a vertical line segment. On the other hand, the initial points on the line $(1-h)(x-$ $1)=b y$ belong to the forbidden set (which is the set of initial conditions that make $z_{n}=-1$ for some $n \geq-1$ ). Thus, we need the line segment $T\left(L_{2}\right)$ to be below $y=$ $\max \{((1-h) / b)(x-1), 1\}$. We achieve this condition by taking $c:=1+b /(1-h)+b^{2} /(1-h)^{2}$. In this way, any initial point $\left(z_{-1}, z_{0}\right) \neq\left(\bar{z}_{2}, \bar{z}_{2}\right)$ that satisfies $z_{-1}>1, z_{0}>c$, or $z_{-1}>c, z_{0}>1$ does not give a positive solution. Hence, the solutions that we are interested in must be within the square $1<x, y<c$. Finally, if $z_{n-1}$ is close to 1 while $z_{n}>1$, then $z_{n+1}=\left(b /\left(z_{n-1}-1\right)\right) z_{n}+h$ will blow up beyond our choice of $c$. Thus, there must be $\epsilon_{0}>0$ such that $z_{n}>1+\epsilon_{0}$ for some fixed $\epsilon_{0}>0$, which completes the proof.

Now, we give the following result.

Theorem 7. Let $0<h<1$. The only solution $\left\{z_{n}\right\}$ of (27) that satisfies $z_{n}>1$ for all $n \geq-1$ is the large equilibrium $\bar{z}_{2}$.

Proof. Suppose that $\left\{z_{n}\right\}$ is a nonequilibrium solution of (27) that satisfies $z_{n}>1$ for all $n \geq-1$. From Lemma 5, we can filter the points that do not obey the monotonicity, and consequently, the sequence $\left\{J_{n}\right\}=\left\{J\left(z_{n-1}, z_{n}\right)\right\}$ has an increasing subsequence $\left\{J_{n_{k}}\right\}$. $\left\{J_{n_{k}}\right\}$ must be bounded by Lemma 6, and, therefore, $\left\{J_{n_{k}}\right\}$ converges to a limit, say, $J_{0}$. On the other hand, the sequence $Z_{n}:=\left(z_{n-1}, z_{n}\right), n=0,1, \ldots$, belongs to a compact set, and therefore, it has a convergent subsequence; say $Z_{n_{j}}=\left(z_{n_{j}-1}, z_{n_{j}}\right), j=0,1, \ldots$, converges to $\left(a_{0}, a_{1}\right)$. Next, we must have $J\left(a_{0}, a_{1}\right)=J_{0}$. Because $\left(\bar{z}_{2}, \bar{z}_{2}\right)$ is a repeller, then $\left(a_{0}, a_{1}\right) \neq\left(\bar{z}_{2}, \bar{z}_{2}\right)$, which together with $J\left(a_{0}, a_{1}\right)=J_{0}$ contradicts Lemma 5 . Hence, a nonequilibrium positive solution cannot stay above one.

Theorem 8. Positive solutions $\left\{z_{n}\right\}_{n=-1}^{\infty}$ of (27) are bounded.

Proof. Let $\left\{z_{n}\right\}_{n=-1}^{\infty}$ be a positive solution of (27). Based on Lemma 6, we need only to handle the case when $z_{n}$ gets between 0 and 1 for some $n=n_{0}$. In this case, we must have $z_{n_{0}+1} \leq(h / b)\left(1-z_{n_{0}}\right)$, because, otherwise, we use the fact that $-1+z_{n_{0}}<0$ to obtain

$$
z_{n_{0}+2}<\frac{b(h / b)\left(1-z_{n_{0}}\right)}{-1+z_{n_{0}}}+h<0 .
$$

Up to this end, we have

$$
\begin{aligned}
& z_{n_{0}+1} \leq \frac{h}{b}\left(1-z_{n_{0}}\right) \leq \frac{h}{b}, \\
& z_{n_{0}+2}=\frac{b z_{n_{0}+1}}{-1+z_{n_{0}}}+h \leq h .
\end{aligned}
$$

Now, an induction argument shows that $z_{n} \leq \max \{h, h / b\}$ for all $n \geq n_{0}+1$, which completes the proof.

Lemma 9. Let $0<h<b<1$. The region bounded by the triangle $\widetilde{\mathscr{D}}_{b, h}$ of vertices $(0,0),(0, h / b)$, and $(h / b, 0)$ forms an invariant of (27). Furthermore, this invariant shrinks under two iterates of $T$ to the invariant $\mathscr{D}_{b, h}$ bounded by the quadrilateral of vertices $(h, 0),(0,0),(0, h)$, and $(h, h(1-b))$.

Proof. Since $h<b<1$, we obtain $h<h / b<1$. Now, consider $(s, t)$ belongs to $\widetilde{\mathscr{D}}_{b, h}$, and then $0 \leq s \leq h / b$ and $0 \leq t \leq h / b-s$. Now,

$$
T(s, t)=\left(t, \frac{b t}{s-1}+h\right),
$$

which belongs to the triangular region $\Delta \subset \widetilde{\mathscr{D}}_{b, h}$ of vertices $(0,0),(0, h)$ and $(h / b, 0)$. Now, let $(s, t) \in \Delta$, and then $0 \leq$ $s \leq h / b$ and $0 \leq t \leq h-b s$, and consequently, $T(s, t)$ and its forward iterates stay inside $\mathscr{D}_{b, h}$. This completes the proof.

3.3. Attractivity of the Small Equilibrium. After establishing an invariant region $\mathscr{D}_{b, h}$ that contains $\bar{z}_{1}$ when $0<h<b<1$, we aim to characterize the behavior of solutions within $\mathscr{D}_{b, h}$. From the fact that

$$
\begin{aligned}
z_{n+1}-\bar{z}_{1}= & \left(z_{n}-\bar{z}_{1}\right) F\left(z_{n-1}\right) \\
& +\bar{z}_{1}\left(F\left(z_{n-1}\right)-F\left(\bar{z}_{1}\right)\right),
\end{aligned}
$$

we observe that if $z_{n-1}, z_{n}>\bar{z}_{1}$ then $z_{n+1}<\bar{z}_{1}$. Similarly, if $z_{n-1}, z_{n}<\bar{z}_{1}$ then $z_{n+1}>\bar{z}_{1}$. Therefore, within $\mathscr{D}_{b, h}$, solutions oscillate about $\bar{z}_{1}$. Consecutive terms of a nonequilibrium solution that satisfy $z_{n} \geq \bar{z}_{1}$ form what we call a positive 
semicycle. Similarly, consecutive terms of a nonequilibrium solution that satisfy $z_{n}<\bar{z}_{1}$ form a negative semicycle. Thus, from (67), we conclude that the length of a semicycle is at most two.

When extrema of consecutive positive (and negative) semicycles form monotonic sequences, we obtain subsequences of the orbit that aids us in characterizing the orbit. This approach has been widely used to prove attractivity of equilibria $[1,11,14,20]$. However, if we follow the extrema of positive semicycles of an orbit of (27), we find it is possible for an extreme value to overshoot (or undershoot) a previous one. For instance, let $h=0.78, b=0.99, z_{-1}=0.06$, and $z_{0}=0.23$. In this case $\bar{z}_{1} \approx 0.3181$, and the orbit through $\left(z_{-1}, z_{0}\right)$ is

$$
\begin{gathered}
\mathcal{O}^{+}\left(z_{-1}, z_{0}\right)=\{0.0600,0.2300, \underline{\mathbf{0 . 5 3 7 8}}, 0.0886, \\
\underline{\mathbf{0 . 5 9 0 3}}, 0.1388, \ldots\},
\end{gathered}
$$

which shows that the extrema of positive semicycles do not form a monotonic sequence.

Instead of bounding an orbit with the extrema of its semicycles, here we develop a technique that bounds the elements of semicycles by a monotonic sequence that does not form a subsequence of the orbit itself, and then we use the monotonic sequence to show the attractivity of $\bar{z}_{1}$. This approach is closely related to the enveloping technique used by $[17,18]$; however, instead of finding one dominant function that goes through the equilibrium, we find a sequence of functions that evolve based on the semicycles of the solution. We write

$$
\begin{aligned}
z_{n+2} & =z_{n+1} F\left(z_{n}\right)+h \\
& =z_{n} F\left(z_{n-1}\right) F\left(z_{n}\right)+h F\left(z_{n}\right)+h,
\end{aligned}
$$

and we define the maps $G_{m}$ as

$$
\begin{aligned}
G_{m}(t) & =\alpha_{m} t|F(t)|+h F(t)+h \\
& =|F(t)|\left(\alpha_{m} t-h\right)+h,
\end{aligned}
$$

in which $\alpha_{m}$ is a fixed value that will be determined by an upper bound of $\left|F\left(z_{n-1}\right)\right|$. In general, we need $G_{m}$ to be increasing with a unique fixed point inside a certain region. Before we embark on our main result, we give some characteristics of $G_{m}$ in the following proposition.

Proposition 10. Consider the function $G_{m}$ as defined in (70) in which $0<h<b<1$ and $F(t)=b /(-1+t)$. Each of the following holds true:

(i) $G_{m}$ is continuous on the interval $[0,1)$.

(ii) $G_{m}(0)=h(1-b)$ and for all $\alpha_{m} \geq h, G_{m}$ is increasing.

(iii) If $\left(b \alpha_{m}-h-1\right)^{2}>4 h(1-b)$, then $G_{m}$ has two fixed points $\bar{u}_{\alpha_{m}}<\bar{v}_{\alpha_{m}}$. In addition, if $\alpha_{m} \leq(1+h) / b$, then $\bar{u}_{\alpha_{m}}$ is increasing while $\bar{v}_{\alpha_{m}}$ is decreasing in $\alpha_{m}$.

Proof. Parts (i) and (ii) are obvious. To verify part (iii), observe that

$$
G_{m}(t)-t=\frac{t^{2}+\left(b \alpha_{m}-h-1\right) t+h(1-b)}{1-t} .
$$

The condition for the existence of two fixed points is obvious, and to obtain $\bar{u}_{\alpha_{m}}$ increasing in $\alpha_{m}$, we must have $b \alpha_{m}-h-1$ negative.

The next result about the attractivity of the small fixed point of $G_{m}$ will be used in the sequel.

Lemma 11. Consider $G_{m}$ as defined in (70), and let $b<1$. If $\alpha_{m}>h$ and

$$
\left(b \alpha_{m}-h-1\right)^{2}>4 h(1-b),
$$

then the equilibrium solution $\bar{u}_{\alpha_{m}}$ of the difference equation $y_{n+1}=G_{m}\left(y_{n}\right)$ is stable with a basin of attraction that contains the interval $\left[0, \bar{v}_{\alpha_{m}}\right)$. Furthermore, the convergence to the equilibrium is monotonic.

Proof. Since $G_{m}(0)>0$ and $G_{m}(t)$ is increasing with two fixed points $\bar{u}_{\alpha_{m}}<\bar{v}_{\alpha_{m}}$, a cobweb (stair-step) diagram shows the result.

Because our goal is to be able to let $\alpha_{m}$ reach $\left|F\left(\bar{z}_{1}\right)\right|$, then taking $\alpha_{m}>\gamma_{r}:=(1 / b)(1+h+2 \sqrt{h(1-b)})$ from the condition $\left(b \alpha_{m}-h-1\right)^{2}>4 h(1-b)$ makes it mandatory to have $\left|F\left(\bar{z}_{1}\right)\right|=\bar{z}_{2}-1>\gamma_{r}$. However, simple computations show that this is not a viable option within our range of parameters. On the other hand, taking $\alpha_{m}<\gamma_{l}:=(1 / b)(1+h-2 \sqrt{h(1-b)})$ makes it necessary for us to have $\bar{z}_{2}-1<\gamma_{l}$, which is indeed a viable option within our range of parameters. Also, since we need $\alpha_{m} \geq h$ to obtain an increasing function $G_{m}$, then we must have $\left|F\left(\bar{z}_{1}\right)\right| \geq h$, However, considering $0<h<b<1$ is sufficient to give $\left|F\left(\bar{z}_{1}\right)\right|=\bar{z}_{2}-1>h$. Thus, to give our approach a chance of success, we need to be accompanied by the following two conditions:

$$
\text { H1: } \begin{aligned}
\bar{z}_{2}-1<\gamma_{l}=\frac{1}{b}(1+h-2 \sqrt{h(1-b)}) \\
\quad\left(\operatorname{so~} \alpha_{m} \text { can reach }\left|F\left(\bar{z}_{1}\right)\right|\right),
\end{aligned}
$$

H2: $0<h<b<1$

$$
\text { ( so } G_{m}(t) \text { is increasing in } t \text { for } 0 \leq t<1 \text { ). }
$$

Next, from the invariant region $\mathscr{D}_{b, h}$ of Lemma 9, we need $|F(h)| \leq \gamma_{l}$ so we can start $\alpha_{1}$ at $|F(h)|$ and then be able to go down to $\left|F\left(\bar{z}_{1}\right)\right|$. Therefore, $|F(h)| \leq \gamma_{l}$ means that we need the condition

$$
\text { H3: } \begin{aligned}
h^{2}+b^{2} \leq 1 & -2(1-h) \sqrt{h(1-b)} \\
& \left(\text { existence, and } \bar{u}_{\alpha_{m}} \uparrow, \bar{v}_{\alpha_{m}} \downarrow \text { in } \alpha_{m}\right) .
\end{aligned}
$$

The next proposition gives the feasible region for the inequalities in $\mathrm{H} 1, \mathrm{H} 2$, and $\mathrm{H} 3$. The proof is just algebraic manipulations of the inequalities.

Proposition 12. The feasible region for the inequalities in $\mathrm{Hl}$, $H 2$, and $H 3$ is the region $\Delta_{b, h}$ bounded by the triangle of vertices $(0,0),(1 / 2,1 / 2),(1,0)$. 
Now, we established enough tools to give the following result.

Theorem 13. Consider (27) with $F(t)=b /(-1+t)$. If $(b, h) \in$ $\Delta_{b, h}$, then all solutions that are attracted to the invariant region $\widehat{\mathscr{D}}_{b, h}$ converge to $\bar{z}_{1}$.

Proof. Based on Lemma 9, since $\widehat{\mathscr{D}}_{b, h}$ shrinks after two iterates of $T$ into $\mathscr{D}_{b, h}$, we can start by $\left(z_{-1}, z_{0}\right) \in \mathscr{D}_{b, h}$. Let $C_{1 l}$ and $C_{1 r}$ be the first negative and positive semicycles (resp.) which belong to the initial condition $\left(z_{-1}, z_{0}\right)$. Let $C_{1}=$ $C_{1 l} \cup C_{1 r}$ denote the first full cycle. Thus, by induction, we consider $C_{m}=C_{m l} \cup C_{m r}$ to be the $m$ th cycle which belongs to the initial condition $\left(z_{-1}, z_{0}\right)$. Now, define $\alpha_{1}:=|F(h)|$, and then by condition $H 3, G_{1}$ has a unique equilibrium, say, $\bar{u}_{\alpha_{1}}$, in the interval $[0, h]$ (the uniqueness follows from the fact that $G_{1}(h)-h \leq 0$ whenever $h \leq 1-b$, which is obtainable from H3). In fact, $0<\bar{u}_{\alpha_{1}} \leq h \leq \bar{v}_{\alpha_{1}}$. Since

$$
z_{n+2}=z_{n}\left|F\left(z_{n}\right)\right|\left|F\left(z_{n-1}\right)\right|+h F\left(z_{n}\right)+h \leq G_{1}\left(z_{n}\right),
$$

we depend on Lemma 11 to conclude that $z_{n+2} \leq \bar{u}_{\alpha_{m}}$ for all elements of the solution passing the first cycle; that is,

$$
z_{n} \leq \bar{u}_{\alpha_{1}} \quad \forall z_{n} \in \bigcup_{j=2}^{\infty} C_{j}
$$

Next, we define $\alpha_{2}=\left|F\left(\bar{u}_{\alpha_{1}}\right)\right|$. Since $\bar{u}_{\alpha_{1}}<h$, we have

$$
\alpha_{2}=\left|F\left(\bar{u}_{\alpha_{1}}\right)\right|<|F(h)|=\alpha_{1} \text {. }
$$

Now, we use the dominant function $G_{2}$ and its unique equilibrium $\bar{u}_{\alpha_{2}}$ in the interval $\left[0, \bar{u}_{\alpha_{1}}\right]$ to conclude again that

$$
z_{n} \leq \bar{u}_{\alpha_{2}} \quad \forall z_{n} \in \bigcup_{j=3}^{\infty} C_{j}
$$

Observe that our function $G_{m}$ evolves in $m$ after each cycle of the oscillatory solution and becomes with a smaller fixed point that dominates the rest of the solution $\left\{z_{n}\right\}$. This induction process follows the cycles of the solution $\left\{z_{n}\right\}$ and keeps giving new upper bounds $\bar{u}_{\alpha_{m}}$ that form a strictly decreasing sequence. Since the sequence $\left\{\bar{u}_{\alpha_{m}}\right\}$ is bounded below by $\left|F\left(\bar{z}_{1}\right)\right|$, consequently, it must converge to a value $\beta \geq\left|F\left(\bar{z}_{1}\right)\right|$. Since $\beta>\left|F\left(\bar{z}_{1}\right)\right|$ can take us to use $\beta$ as a new starting point and repeat the same process again, we find that the viable option is to take $\beta:=\left|F\left(\bar{z}_{1}\right)\right|$. Therefore, we conclude that $\lim \sup z_{n} \leq \bar{z}_{1}$, and because our solution $\left\{z_{n}\right\}$ is oscillatory inside the region $\mathscr{D}_{b, h}$, then we must have

$$
\lim \sup z_{n}=\liminf z_{n}=\bar{z}_{1} \text {, }
$$

which completes the proof.

We close this section by the following remark.

Remark 14. It is worth mentioning that the method of this section can be developed under more general settings to handle a wider class of maps $F$. Also, we remark that relaxing the condition $0<h<b<1$ adds another factor of complexity to the problem because periodic solutions of period two and three come to the scene. For instance, when $h=1-b(1-b)$, the period-three solution $\{0, h, h(1-b)\}$ is located on the boundary of the region $\mathscr{D}_{b, h}$.

\section{Conclusion and Discussion}

In this paper, we considered the difference equation

$$
z_{n+1}=\frac{b z_{n}}{-1+z_{n-1}}+h, \quad b, h>0,
$$

which can be considered a prototype for the more general form $z_{n+1}=z_{n} F\left(z_{n-1}\right)+h$ where $F(z)$ and $z F(z)$ are both decreasing. For $h>1$, we established a connection between (81) and the well-known Y2K difference equation. Proving global stability in the $\mathrm{Y} 2 \mathrm{~K}$ was settled by Merino in [19]. However, we gave a new proof of the crucial lemma used by Merino to prove the global stability of the positive equilibrium with respect to the positive quadrant.

For (the new range of parameters) $0<h<1$, we restricted our investigation to nonnegative solutions that exist for all $n \geq-1$, and we showed that such solutions (except the large equilibrium which is a repeller) eventually go below one. This fact makes all nonnegative solutions bounded. We found subregions of the basin of attraction and gave conditions under which nonnegative solutions converge to the stable equilibrium $\bar{z}_{1}$.

Several aspects of solutions of (81) remain unexplored and worth further investigation in future work, to name a few, identifying the forbidden set, finding the exact basin of attraction of $\bar{z}_{1}$, and investigating the behaviour of solutions that take negative values. Finally, it is worth stressing that the choice of the function $F(z)$ serves as an entry point for future exploration of the difference equation $z_{n+1}=z_{n} F\left(z_{n+1}\right)+h$ when $z F(z)$ is decreasing.

\section{Conflict of Interests}

The authors declare that there is no conflict of interests regarding the publication of this paper.

\section{Acknowledgment}

The authors would like to thank Nasser Al-Salti for the stimulating discussion they had while working on the problem.

\section{References}

[1] V. L. Kocic and G. Ladas, Global Behavior of Nonlinear Difference Equations of Higher Order with Applications, Kluwer Academic Publishers, Dordrecht, The Netherlands, 1993.

[2] J. D. Murray, Mathematical Biology, Springer, New York, NY, USA, 1989.

[3] L. D. Mueller and A. Joshi, Stability in Model Populations, Princeton University Press, Princeton, NJ, USA, 2000.

[4] E. C. Pielou, Population and Community Ecology, Gordon and Breach, New York, NY, USA, 1974. 
[5] S. A. Levin and R. M. May, "A note on difference-delay equations," Theoretical Population Biology, vol. 9, no. 2, pp. 178-187, 1976.

[6] E. Liz, V. Tkachenko, and S. Trofimchuk, "Global stability in discrete population models with delayed-density dependence," Mathematical Biosciences, vol. 199, no. 1, pp. 26-37, 2006.

[7] R. Abu-Saris, Z. AlSharawi, and M. B. Rhouma, "The dynamics of some discrete models with delay under the effect of constant yield harvesting," Chaos, Solitons \& Fractals, vol. 54, pp. 26-38, 2013.

[8] Z. AlSharawi, "A global attractor in some discrete contest competition models with delay under the effect of periodic stocking," Abstract and Applied Analysis, vol. 2013, Article ID 101649, 7 pages, 2013.

[9] Z. AlSharawi and M. B. Rhouma, "The Beverton-Holt model with periodic and conditional harvesting," Journal of Biological Dynamics, vol. 3, no. 5, pp. 463-478, 2009.

[10] L. Arriola, "First integrals for difference equations," Nonlinear Analysis: Theory, Methods \& Applications, vol. 30, pp. 1191-1196, 1997.

[11] E. A. Grove and G. Ladas, Periodicities in Nonlinear Difference Equations, CRC Press, Boca Raton, Fla, USA, 2000.

[12] E. A. Grove, V. L. Kocic, and G. Ladas, "Classification of invariants for certain difference equations," in Advances in Difference Equations: Proceedings of the Second International Conference on Difference Equations, Hungary, 1995, Gordon and Breach, 1997.

[13] T. Nesemann, "Invariants and Liapunov functions for nonautonomous systems," Computers \& Mathematics with Applications, vol. 42, no. 3-5, pp. 385-392, 2001.

[14] E. Camouzis and G. Ladas, Dynamics of Third-Order Rational Difference Equations with Open Problems and Conjectures, Chapman \& Hall, CRC Press, 2008.

[15] R. D. Nussbaum, "Global stability, two conjectures and Maple," Nonlinear Analysis: Theory, Methods \& Applications, vol. 66, no. 5, pp. 1064-1090, 2007.

[16] P. Cull, "Enveloping implies global stability," in Difference Equations and Discrete Dynamical Systems (Proceedings of the 9th Annual International Conference on Difference Equations and Applications, Los Angeles, Calif, USA, 2004), pp. 71-85, World Science Publisher, 2005.

[17] H. A. El-Morshedy and V. J. López, "Global attractors for difference equations dominated by one-dimensional maps," Journal of Difference Equations and Applications, vol. 14, no. 4, pp. 391-410, 2008.

[18] V. J. Lopez, “The Y2K problem revisited," Journal of Difference Equations and Applications, vol. 16, no. 1, pp. 105-119, 2010.

[19] O. Merino, "Global attractivity of the equilibrium of a difference equation: an elementary proof assisted by computer algebra system," Journal of Difference Equations and Applications, vol. 17, no. 1, pp. 33-41, 2011.

[20] M. R. Kulenovic and G. Ladas, Dynamics of Second Order Rational Difference Equations, Chapman and Hall/CRC, Boca Raton, Fla, USA, 2002. 


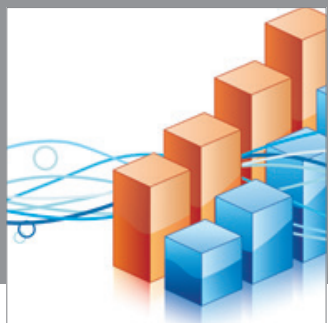

Advances in

Operations Research

mansans

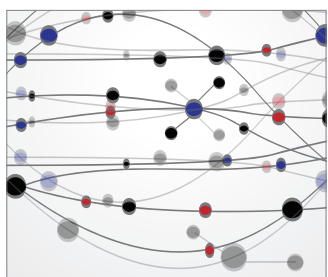

The Scientific World Journal
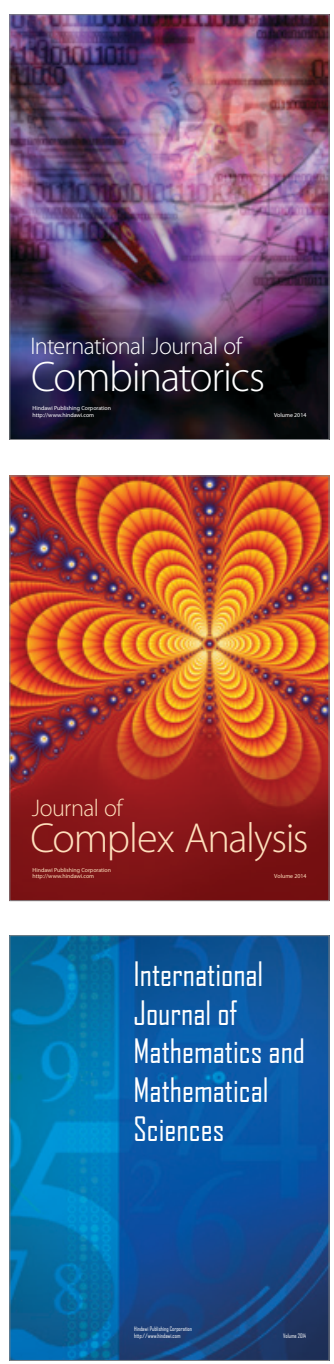
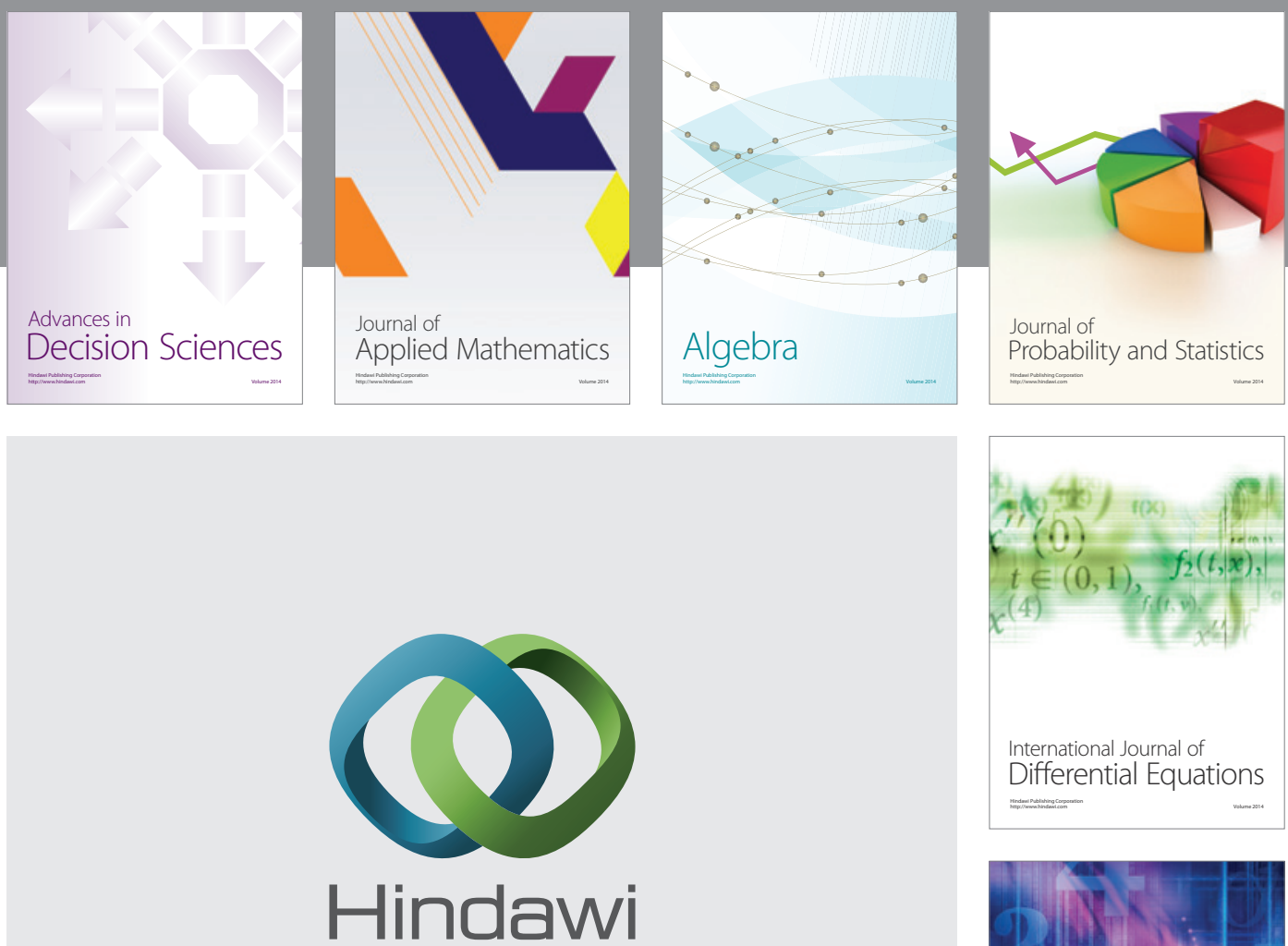

Submit your manuscripts at http://www.hindawi.com
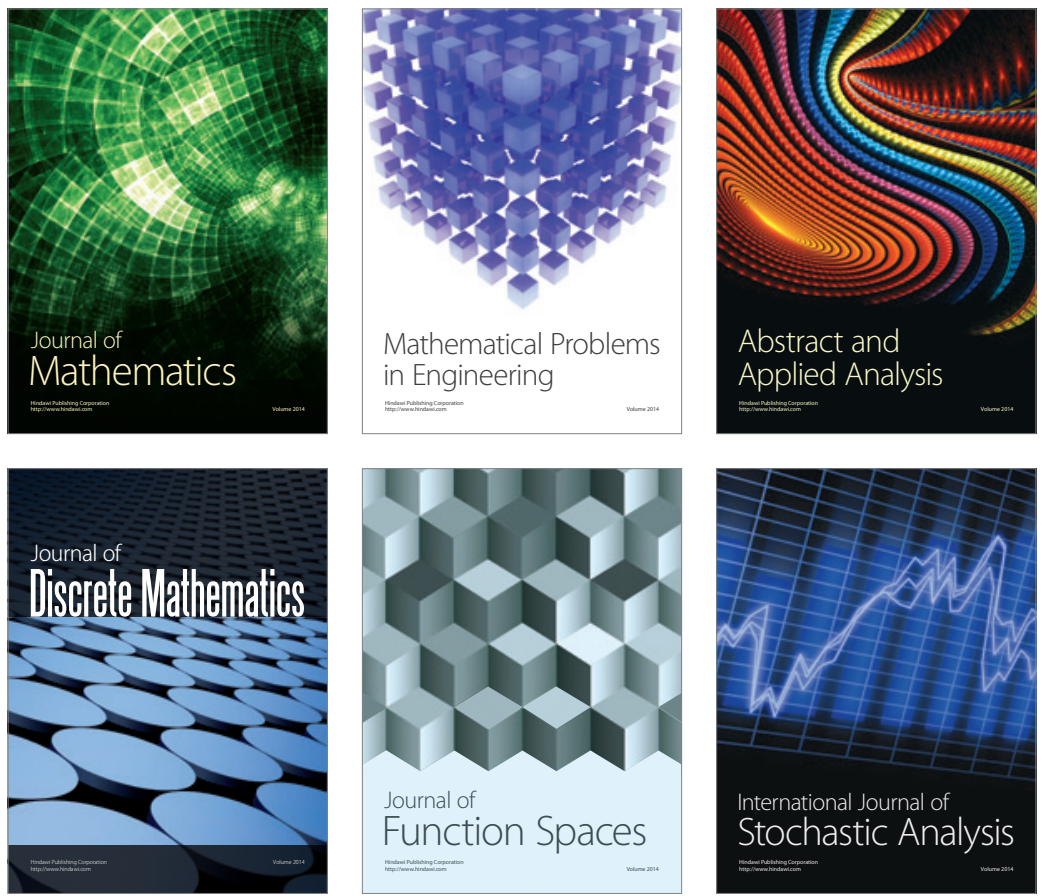

Journal of

Function Spaces

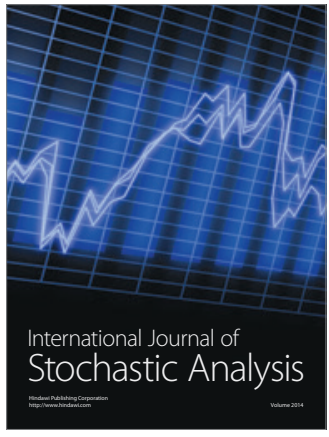

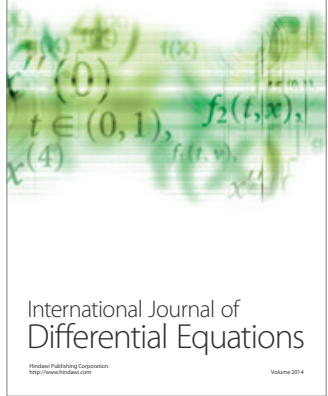
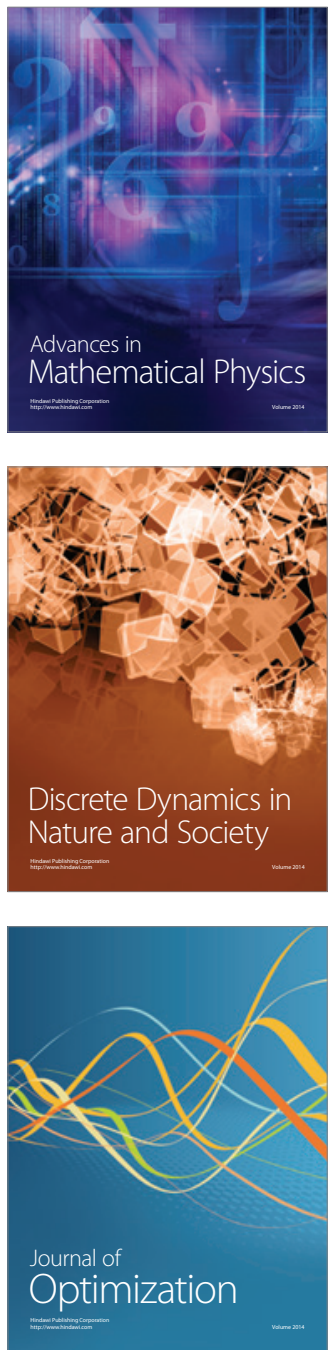